\title{
ON PRIMES AND PRACTICAL NUMBERS
}

\author{
CARL POMERANCE AND ANDREAS WEINGARTNER
}

\begin{abstract}
A number $n$ is practical if every integer in $[1, n]$ can be expressed as a subset sum of the positive divisors of $n$. We consider the distribution of practical numbers that are also shifted primes, improving a theorem of Guo and Weingartner. In addition, essentially proving a conjecture of Margenstern, we show that all large odd numbers are the sum of a prime and a practical number. We also consider an analogue of the prime $k$-tuples conjecture for practical numbers, proving the "correct" upper bound, and for pairs, improving on a lower bound of Melfi.
\end{abstract}

\author{
In memory of Ron Graham (1935-2020) \\ and Richard Guy (1916-2020)
}

\section{INTRODUCTION}

After Srinivasan [16], we say a positive integer $n$ is practical if every integer $m \in[1, n]$ is a subset-sum of the positive divisors of $n$. After the proof of Erdős [2] in 1950 that the practical numbers have asymptotic density 0 , their distribution has been of some interest, with work of Margenstern, Melfi, Tenenbaum, Saias, and the second-named author of this paper. In particular, we now know, 23], 24, that there is a constant $c=1.33607 \ldots$ such that the number of practical numbers in $[1, x]$ is $\sim c x / \log x$ as $x \rightarrow \infty$. For other problems and results about practical numbers see [5, Sec. B2].

The problem of how frequently a shifted prime $p-h$ can be practical was considered recently in [4. Since practical numbers larger than 1 are all even, one assumes that the shift $h$ is a fixed odd integer. Under this assumption, it would make sense that the concept of being practical and being a shifted prime are "independent events" and so it is natural to conjecture that the number of primes $p \leq x$ with $p-h$ practical is of magnitude $x / \log ^{2} x$. Towards this conjecture it was shown in [4 that the number of shifted primes up to $x$ that are practical is, for large $x$

Date: October 27, 2020.

2000 Mathematics Subject Classification. 11N25 (11N37).

Key words and phrases. practical number, shifted prime. 
depending on $h$, between

$$
\frac{x}{(\log x)^{5.7683 \ldots}} \text { and } \frac{x}{(\log x)^{1.0860 \ldots}} \text {. }
$$

Here we make further progress with this problem, proving the conjecture for the upper bound of the count and reducing the lower bound exponent $5.7683 \ldots$ to $3.1647 \ldots$.

As in 4] we consider a somewhat more general problem. Let $\theta$ be an arithmetic function with $\theta(n) \geq 2$ for all $n$ and let $\mathcal{B}_{\theta}$ be the set of positive integers containing $n=1$ and all those $n \geq 2$ with canonical prime factorization $n=p_{1}^{\alpha_{1}} p_{2}^{\alpha_{2}} \cdots p_{k}^{\alpha_{k}}, p_{1}<\ldots<p_{k}, \alpha_{1}, \ldots, \alpha_{k} \geq 1$, which satisfy

$$
p_{j} \leq \theta\left(p_{1}^{\alpha_{1}} \ldots p_{j-1}^{\alpha_{j-1}}\right) \quad(1 \leq j \leq k) .
$$

(It is not necessary that $p_{i}$ be the $i$-th prime number.) Stewart [17] and Sierpinski [15] showed that if $\theta(n)=\sigma(n)+1$, where $\sigma(n)$ is the sum of the positive divisors of $n$, then the set $\mathcal{B}_{\theta}$ is precisely the set of practical numbers. Tenenbaum [20] found that if $\theta(n)=y n$, where $y \geq 2$ is a constant, then $\mathcal{B}_{\theta}$ is the set of integers with $y$-dense divisors; i.e., the ratios of consecutive divisors are at most $y$.

Throughout this paper, all constants implied by the big $O$ and $\ll$ notation may depend on the choice of $\theta$. For several of our results we assume that there are constants $A, C$ such that

$$
\theta(m n) \leq C m^{A} \theta(n), \quad m, n \geq 1 .
$$

This holds for $\theta(n)=\sigma(n)+1$ with $A=2, C=1$, since we trivially have $\sigma(m n) \leq \sigma(m) \sigma(n)$ and $\sigma(m) \leq m^{2}$.

We write $\log _{2} x=\log \log x$ for $x>e^{e}$ and $\log _{2} x=1$ for $0<x \leq e^{e}$, and write $\log _{3} x=\log _{2} \log x$ for $x>1$. Let

$$
l(x)=\exp \left(\frac{\log x}{\log _{2} x \log _{3}^{3} x}\right)
$$

and

$$
S_{h}(x):=\mid\left\{p \leq x: p \text { prime, } p-h \in \mathcal{B}_{\theta}\right\} \mid .
$$

Theorem 1. Fix a nonzero integer $h$. Assume (2) and $n \leq \theta(n) \ll$ $n l(n)$ for $n \geq 1$. For $x$ sufficiently large depending on the choice of $\theta, h$, we have

$$
\frac{x}{(\log x)^{3.1648}}<S_{h}(x) \ll_{h} \frac{x}{(\log x)^{2}},
$$

where $h \in \mathbb{Z}$ and $h$ is not divisible by $\prod_{p \leq \theta(1)} p$ in the lower bound. 
The exponent in the lower bound can be taken as any number larger than $(e+1) \log (e+1)-e+1$. In the case of practical numbers, where $\theta(n)=\sigma(n)+1$ and $\prod_{p \leq \theta(1)} p=2$, Theorem 1 implies the following.

Corollary 1. For any fixed odd $h \in \mathbb{Z}$, the number of primes $p \leq x$ such that $p-h$ is practical satisfies (3).

It seems likely that the upper bound in (3) is best possible, apart from optimizing the implied constant as a function of the shift parameter $h$. Our proof shows that this constant is $\ll h / \varphi(h)$.

Margenstern [8, Conjecture 7] conjectured that every natural number other than 1 is the sum of two numbers that are either practical or prime. The case of even numbers was settled by Melfi [10, Theorem $1]$, who showed that every even number is the sum of two practical numbers. Somewhat weaker versions of the problem for odd numbers were recently stated by Sun [18]. (Also see [19] for several other related problems.) We show that, in the case of odd numbers, there are at most a finite number of exceptions to Margenstern's conjecture. Tomás Oliveira e Silva has told us that Margenstern's conjecture has no counterexamples to $10^{9}$ and we have verified this via a direct search. We have used this result to bootstrap the calculation to a considerably higher bound, see Section 5. It may be difficult by our methods to get a numerical bound $x_{0}$ for which every odd number $>x_{0}$ is the sum of a prime and a practical number, but such a calculation is tractable using our proof if one is prepared to use the extended Riemann Hypothesis in place of the Bombieri-Vinogradov theorem. However, it may be that even this hypothetical $x_{0}$ is too large for a feasible calculation to close the gap.

Theorem 2. Assume $\theta(n) \geq n$. Every sufficiently large integer not divisible by $\prod_{p \leq \theta(1)} p$ is the sum of a prime and a member of $\mathcal{B}_{\theta}$.

Corollary 2. Every sufficiently large odd integer is the sum of a prime and a practical number.

Margenstern [8, Theorem 6] showed that for every fixed even number $h$, there are infinitely many practical numbers $n$ such that $n+h$ is also practical. He conjectured [8, Conjecture 2] that the number of practical pairs $\{n, n+2\}$ up to $x$ is asymptotic to $c x / \log ^{2} x$ for some positive constant $c$. Let

$$
T_{h}(x):=\left|\left\{n \leq x: n \in \mathcal{B}_{\theta}, n+h \in \mathcal{B}_{\theta}\right\}\right| .
$$

Theorem 3. Fix a nonzero integer $h$. Assume (2) and $\theta(n) \ll n l(n)$ for $n \geq 1$. 
(i) We have

$$
T_{h}(x) \ll_{h} \frac{x}{\log ^{2} x} .
$$

(ii) Assume further that $\theta(n) \geq n$ for all $n$, and that $n \in \mathcal{B}_{\theta}$ and $m \leq 3 n /|h|$ imply $m n \in \mathcal{B}_{\theta}$. Moreover, if $\theta(1)<3$, assume that

$$
\begin{cases}h \in 2 \mathbb{Z} & \text { if } \theta(2) \geq 3, \\ h \in 4 \mathbb{Z} & \text { if } \theta(2)<3 .\end{cases}
$$

Then for sufficiently large $x$, depending on the choice of $h$,

$$
T_{h}(x)>\frac{x}{(\log x)^{9.5367}} .
$$

When $h \in 2 \mathbb{Z}$ and $\theta(n)=\sigma(n)+1$, all conditions of Theorem 3 are satisfied, since for practical $n$ we have $\sigma(n)+1 \geq 2 n$, by [8, Lemma 2].

Corollary 3. For every nonzero even integer $h$, the number of practical $n$ up to $x$, such that $n+h$ is also practical, satisfies (41) and (6).

Corollary 3 improves on the lower bound by Melfi [11, Thm. 1.1] for twin practical numbers, $T_{2}(x) \gg x / \exp (k \sqrt{\log x})$ for $k>2+\log (3 / 2)$.

The upper bound in Theorem 3 generalizes as follows to the distribution of practical $k$-tuples.

Theorem 4. Fix integers $0 \leq h_{1}<h_{2}<\ldots<h_{k}$. Assume (2) and $\theta(n) \ll n l(n)$ for $n \geq 1$. We have

$$
\left|\left\{n \leq x:\left\{n+h_{1}, \ldots, n+h_{k}\right\} \subset \mathcal{B}_{\theta}\right\}\right| \ll_{h_{1}, \ldots, h_{k}} \frac{x}{\log ^{k} x} .
$$

When $k \geq 3$ getting a lower bound of the same quality for these $k$-tuples seems difficult. In some cases with the practical numbers we know there are no large examples, such as when the $h_{i}$ do not all have the same parity, or for the example $0,2,4,6$ when at least one of $n+h_{i}$ must be $2(\bmod 4)$ and not divisible by 3, cf. [8]. However, when the $k$-tuple is admissible, i.e., not ruled out by congruence conditions, it would seem likely that the "independent events" heuristic would again apply and that the upper bound in Theorem 4 is correct up to a constant factor. In our proof of the lower bound in Theorem 3 we use the Bombieri-Vinogradov theorem. If instead the Elliott-Halberstam conjecture is assumed, it may be possible to get a reasonable lower bound in Theorem 4 when the $k$-tuple is admissible in the sense above. Finally, we remark that in certain special cases, such as when the $h_{i}$ are $0,2,4$, we at least know that there are infinitely many practical examples, see Melfi [10]. 


\section{The Upper Bound of Theorem 1}

Lemma 1. There exists a constant $K>0$ such that for all $a, b \in \mathbb{Z} \backslash\{0\}$ and all $x>1$ we have

$$
\mid\{m \leq x: m \text { and } a m+b \text { are both prime }\} \mid \leq K \frac{a|b|}{\varphi(a|b|)} \cdot \frac{x}{\log ^{2} x} .
$$

This result follows immediately from [12, Lemma 5].

Let $P^{+}(n)$ denote the largest prime factor of $n>1$ and $P^{+}(1)=1$. Define

$$
B(x, y, z)=\left|\left\{n \leq x: n \in \mathcal{B}_{z \theta}, P^{+}(n) \leq y\right\}\right| .
$$

Proposition 1. Assume $\theta(n) \ll n l(n)$. For $x \geq 2$, $y \geq 2$ and $z \geq 1$,

$$
B(x, y, z) \ll \frac{x \log (2 z)}{\log x} e^{-u / 3},
$$

where $u=\log x / \log y$.

Before proving this we establish some consequences.

Corollary 4. Let $\alpha \in \mathbb{R}$. Assume (2) and $\theta(n) \ll n l(n)$ for $n \geq 1$. For $x \geq 1, y \geq 2, z \geq 1$,

$$
\sum_{\substack{n \leq x, n \in \mathcal{B}_{z \theta} \\ P^{+}(n) \leq y}}\left(\frac{\sigma(n)}{n}\right)^{\alpha} \ll_{\alpha} \frac{x \log (2 z)}{\log (2 x)} \exp \left(-\frac{\log x}{3 \log y}\right) .
$$

Proof. When $\alpha \leq 0$, the result follows from Proposition 1. We will show the result for $\alpha \in \mathbb{N}$ by induction. Note that because of (2) we have that $k d \in \mathcal{B}_{\theta}$ implies $k \in \mathcal{B}_{\theta_{d}}$, where $\theta_{d}(n)=C d^{A} \theta(n)$. By Proposition 11 with $z$ replaced by $z C d^{A}$,

$$
\begin{aligned}
\sum_{\substack{n \leq x, n \in \mathcal{B}_{z \theta} \\
P+(n) \leq y}} & \left(\frac{\sigma(n)}{n}\right)^{\alpha}=\sum_{\substack{n \leq x, n \in \mathcal{B}_{z \theta} \\
P^{+(n) \leq y}}}\left(\frac{\sigma(n)}{n}\right)^{\alpha-1} \sum_{d \mid n} \frac{1}{d} \\
& \leq \sum_{d \leq x} \frac{\sigma(d)^{\alpha-1}}{d^{\alpha}} \sum_{\substack{k \leq x / d, k \in \mathcal{B}_{z \theta} \\
P^{+}(k) \leq y}}\left(\frac{\sigma(k)}{k}\right)^{\alpha-1} \\
& \ll \alpha \sum_{d \leq x} \frac{\sigma(d)^{\alpha-1}}{d^{\alpha}} \frac{x \log (2 d z)}{d \log (2 x / d)} \exp \left(-\frac{\log (x / d)}{3 \log y}\right) \\
& \ll x \exp \left(-\frac{\log x}{3 \log y}\right) \sum_{d \leq x} \exp \left(\frac{\log d}{3 \log y}\right) \frac{\left(\log _{2} d\right)^{\alpha-1} \log (2 d z)}{d^{2} \log (2 x / d)} \\
& \ll \alpha \frac{x \log (2 z)}{\log (2 x)} \exp \left(-\frac{\log x}{3 \log y}\right)
\end{aligned}
$$


since $\exp ((\log d) /(3 \log y)) \leq d^{1 / 2}$.

With $y=x, z=1$ and $\alpha=1$ in Corollary 4 , we get

Corollary 5. Under the assumptions of Corollary 4 we have, for $x>1$,

$$
\sum_{\substack{n \leq x \\ n \in \mathcal{B}_{\theta}}} \frac{\sigma(n)}{n} \ll \frac{x}{\log x} .
$$

Remark 1. Corollary 5 allows us to replace the relative error term $O\left(\log _{2} x / \log x\right)$ in [23, Theorem 1.1], the asymptotic for the count of practical numbers up to $x$, by $O(1 / \log x)$. Indeed, in the proof of [23, Theorem 1.1], the estimate $\sigma(n) / n \ll \log _{2} n$ leads to the extra factor of $\log _{2} x$. Using instead Corollary 5 in the proofs of Lemmas 5.3 and 5.6 of [23], the factor $\log _{2} x$ can be avoided.

Proof of the upper bound in Theorem 1. Assume $x \geq 2|h|$. We consider those $n \in \mathcal{B}_{\theta}$ with $n+h$ prime and $n+h \leq x$. We may assume that $n>x / \log ^{2} x$. Write $n=m q$, where $q=P^{+}(n)$. We have $m \in \mathcal{B}_{\theta}$, $P^{+}(m) \leq q$ and $q \leq \theta(m) \leq m l(m)$. So, assuming $x$ is large, we have $m>x^{1 / 3}$. By Lemma 1 ,

$$
\begin{aligned}
S_{h}(x) & \leq \sum_{m \in \mathcal{B}_{\theta}} \mid\{q \text { prime }: m q+h \text { prime }, q \leq(x-h) / m\} \mid \\
& \ll \sum_{\substack{m \in \mathcal{B}_{\theta}, m>x^{1 / 3} \\
m P^{+}(m) \leq x-h}} \frac{m|h|}{\varphi(m|h|)} \frac{(x-h) / m}{\log ^{2}(2(x-h) / m)} \\
& \leq \frac{2|h| x}{\varphi(|h|)} \sum_{m \in \mathcal{B}_{\theta}, m>x^{1 / 3}} \frac{1}{\varphi(m) \log ^{2} P^{+}(m)} .
\end{aligned}
$$

We will show that the last sum is $\ll 1 / \log ^{2} x$. With $p=P^{+}(m)$ and $m=k p$, we have $k \in \mathcal{B}_{\theta}$ and $k>x^{1 / 7}$. The last sum is

$$
\ll \sum_{p \geq 2} \frac{1}{p \log ^{2} p} \sum_{\substack{k \in \mathcal{B}_{\theta}, k>x^{1 / 7} \\ P+(k) \leq p}} \frac{k}{\varphi(k)} \cdot \frac{1}{k} .
$$

Since $k / \varphi(k) \ll \sigma(k) / k$, Corollary 4 (with $\alpha=z=1$ ) and partial summation applied to the inner sum shows that the last expression is

$$
\ll \sum_{p \geq 2} \frac{1}{p \log ^{2} p} \cdot \frac{\log p}{\log x} \exp \left(-\frac{\log x}{21 \log p}\right) \ll \frac{1}{\log ^{2} x},
$$

by the prime number theorem. 
Proof of Proposition 11. We follow the proof of Saias [13, Prop. 1], who established this result in the case when $\theta(n)=y n$ with $y \geq 2$ (integers with $y$-dense divisors) and in the case when $\theta(n)=\sigma(n)+1$ (practical numbers) and $z=1$. Let $f(n)$ be an increasing function with $\theta(n) \leq n f(n)$ for all $n \geq 1$ and $f(n) \ll l(n)$. Suppose $n \in \mathcal{B}_{z \theta}$, where $n=p_{1} p_{2} \ldots p_{k}$ with $p_{1} \leq p_{2} \leq \cdots \leq p_{k}$. Since $f$ is increasing, $p_{j} \leq z p_{1} \cdots p_{j-1} f\left(p_{1} \cdots p_{j-1}\right)$, so $p_{j}^{2} \leq z n f(n) \leq z x f(x)$ for $n \leq x$. By sorting the integers counted in $B(x, y, z)$ according to their largest prime factor, we get

$$
B(x, y, z) \leq 1+\sum_{p \leq \min (y, \sqrt{z x f(x)})} B(x / p, p, z),
$$

the analogue of [13, Lemma 8].

Let $\Psi(x, y)$ denote the number of integers $n \leq x$ with $P^{+}(n) \leq y$. We write $u=\log x / \log y$ and $\tilde{v}=\log x / \log (2 z)$. Let $\tilde{\rho}(u)=\rho(\max \{0, u\})$, where $\rho(u)$ is Dickman's function. Let $\tilde{D}(x, y, z)$ be the function defined in [13, p. 169]. It satisfies

$$
\tilde{D}(x, y, 2 z) \asymp \frac{x}{\tilde{v}} \tilde{\rho}(u(1-1 / \sqrt{\log y})-1) \quad\left(0<u<3(\log x)^{1 / 3}\right)
$$

and

$$
\tilde{D}(x, y, 2 z)=\Psi(x, y) \quad\left(u \geq 3(\log x)^{1 / 3}\right),
$$

Lemma 9 of [13] shows that

$$
\tilde{D}(x, y, 2 z) \geq 1+\sum_{p \leq \min (y, \sqrt{2 z x} l(x))} \tilde{D}(x / p, p, 2 z),
$$

for $z \geq 1, y \geq 2, \tilde{v} \geq v_{0}$ and $0<u \leq 3(\log x)^{1 / 3}$.

We claim that

$$
B(x, y, z) \leq c \tilde{D}(x, y, 2 z)
$$

for some suitable constant $c$. If $2 \leq x \leq x_{0}$, we have $\tilde{D}(x, y, 2 z) \asymp 1$, so we may assume $x \geq x_{0}$ and hence $\sqrt{f(x)} \leq l(x)$. If $0<\tilde{v} \leq$ $u<3(\log x)^{1 / 3}$, then $2 z \geq y$ and $B(x, y, z)=\Psi(x, y) \ll \tilde{D}(x, y, 2 z)$, where the last estimate is derived in the penultimate display on page 182 of [13]. If $0<u \leq \tilde{v} \leq v_{0}$, then $\tilde{D}(x, y, 2 z) \asymp x$ so (7) holds. If $u \geq 3(\log x)^{1 / 3}$, then $\tilde{D}(x, y, 2 z)=\Psi(x, y)$ and (7) holds. Assume that $c$ is such that (7) holds in the domain covered so far. In the remainder we may assume that $u \leq 3(\log x)^{1 / 3}$ and $\tilde{v} \geq v_{0}$. We show by induction 
on $k$ that (7) holds for $y \geq 2, z \geq 1,2 \leq x \leq 2^{k}$. We have

$$
\begin{aligned}
B(x, y, z) & \leq 1+\sum_{p \leq \min (y, \sqrt{z x f(x)})} B(x / p, p, z) \\
& \leq 1+c \sum_{p \leq \min (y, \sqrt{z x f(x)})} \tilde{D}(x / p, p, 2 z) \\
& \leq c\left(1+\sum_{p \leq \min (y, \sqrt{2 z x} l(x))} \tilde{D}(x / p, p, 2 z)\right) \\
& \leq c \tilde{D}(x, y, 2 z) .
\end{aligned}
$$

It remains to show that

$$
\tilde{D}(x, y, 2 z) \ll x \frac{\log (2 z)}{\log x} e^{-u / 3} .
$$

We may assume $x \geq x_{0}$. If $u \leq 3(\log x)^{1 / 3}$, then $y \geq y_{0}$ and the result follows from $\rho(u) \ll e^{-u}$. If $u>3(\log x)^{1 / 3}$, then

$$
\tilde{D}(x, y, 2 z)=\Psi(x, y) \ll x e^{-u / 2} \ll \frac{x}{\log x} e^{-u / 3} \ll \frac{x \log (2 z)}{\log x} e^{-u / 3},
$$

where the upper bound for $\Psi(x, y)$ is [21, Thm. III.5.1].

\section{Some Lemmas}

The following observation follows immediately from the definition of the set $\mathcal{B}_{\theta}$ in (1).

Lemma 2. Let $\theta(n) \geq n$ for all $n \in \mathbb{N}$. If $n \in \mathcal{B}_{\theta}$ and $P^{+}(k) \leq n$, then $n k \in \mathcal{B}_{\theta}$.

If $\theta(n)=y n$, we write $\mathcal{D}_{y}$ for $\mathcal{B}_{\theta}$. For an integer $n>1$, let $P^{-}(n)$ denote the least prime dividing $n$, and let $P^{-}(1)=+\infty$.

Lemma 3. There is a number $y_{0}$ such that if $x \geq z^{4} \geq 1$ and $y \geq$ $\max \left\{y_{0}, z+z^{0.535}\right\}$, we have

$$
\left|\left\{n \leq x: n \in \mathcal{D}_{y}, P^{-}(n)>z\right\}\right| \asymp \frac{x \log (y / z)}{\log (x y) \log (2 z)} .
$$

This conclusion continues to hold if $z+1 \leq y \leq y_{0}$ and $(z, y]$ contains at least one prime number.

Proof. When $x \geq y \geq y_{0}$ and $z \geq 3 / 2$, then $\log (x y) \asymp \log x$ and the result follows from [14, Thm. 1] and [22, Rem. 2]. When $y>x$, the result follows from $\left|\left\{n \leq x: P^{-}(n)>z\right\}\right| \asymp x / \log (2 z)$. If $1 \leq z \leq 3 / 2$, 
the result follows from [13, Thm. 1]. If $y \leq y_{0}$, the result follows from iterating [14, Lemma 8] a finite number of times.

Lemma 4. For $d \in \mathbb{N}, x \geq 1, z \geq 1$ and $y \geq 2 z$, we have

$$
\left|\left\{n \leq x: n \in \mathcal{D}_{y}, P^{-}(n)>z, d \mid n\right\}\right| \ll 1_{d \in \mathcal{D}_{y}}+\frac{x \log (d y)}{d \log (x y) \log (2 z)} .
$$

Proof. We first assume that $x / d \geq z^{4}$. If $d=1$ the result follows from Lemma 3, so we assume $d>1$. We have

$$
\begin{aligned}
\left|\left\{d w \leq x: d w \in \mathcal{D}_{y}, P^{-}(w)>z\right\}\right| & \leq\left|\left\{w \leq x / d: w \in \mathcal{D}_{d y}, P^{-}(w)>z\right\}\right| \\
& \ll \frac{x \log (d y)}{d \log (x y) \log (2 z)},
\end{aligned}
$$

by Lemma 3 .

If $x / d \leq z^{4}$, then $\log (x y) \leq \log \left(y d z^{4}\right) \leq 5 \log (y d)$, so the result follows from $\left|\left\{2 \leq w \leq x / d: P^{-}(w)>z\right\}\right| \ll x /(d \log (2 z))$.

Lemma 5. Assume $\theta(n) \geq n$ for all $n \in \mathbb{N}$. For all $h \in \mathbb{N}$ that are not divisible by $\prod_{p \leq \theta(1)} p$, we have

$$
\left|\left\{x / p_{0}<n \leq x: n \in \mathcal{B}_{\theta}, \operatorname{gcd}(n, h)=1\right\}\right| \gg \frac{x}{\log x \log (2 h) \log _{2} h},
$$

for $x \geq K \log ^{5}(2 h)$, where $p_{0} \leq \theta(1)$ is the smallest prime not dividing $h$, and $K$ is some positive constant depending only on $\theta$. Moreover, there exists a constant $\eta>0$ such that if $L \geq 1$ satisfies

$$
\sum_{p \mid h, p>L} \frac{\log p}{p}<\eta
$$

then, for $x \geq K L^{5}$,

$$
\left|\left\{x / p_{0}<n \leq x: n \in \mathcal{B}_{\theta}, \operatorname{gcd}(n, h)=1\right\}\right| \gg \frac{x}{L \log x \log (2 L)} .
$$

Proof. Let $p_{0} \leq \theta(1)$ be the smallest prime with $p_{0} \nmid h$. Let $k \in \mathbb{N}$, $L_{k}=p_{0}^{k} / 2$, and assume $x \geq 2 L_{k}^{5}$. Since $\theta(n) \geq n$,

$$
\begin{aligned}
\mid\left\{x / p_{0}<n=p_{0}^{k} w \leq x\right. & \left.: n \in \mathcal{B}_{\theta}, P^{-}(w)>L_{k}\right\} \mid \\
& \geq\left|\left\{x / p_{0}^{k+1}<w \leq x / p_{0}^{k}: w \in \mathcal{D}_{p_{0}^{k}}, P^{-}(w)>L_{k}\right\}\right| .
\end{aligned}
$$

We would like to use Lemma 3 to obtain a lower bound for this count, but the fact that $w$ is not free to roam over the entire interval $\left[1, x / p_{0}^{k}\right]$ is problematic. We note though that Lemma 3 implies there is a set 
$\mathcal{K} \subset \mathbb{N}$ with bounded gaps such that if $x \geq 2 L_{k}^{5}$ and $k \in \mathcal{K}$, we have

$$
\begin{aligned}
\left|\left\{x / p_{0}^{k+1}<w \leq x / p_{0}^{k}: w \in \mathcal{D}_{p_{0}^{k}}, P^{-}(w)>L_{k}\right\}\right| & \gg \frac{x \log \left(p_{0}^{k} / L_{k}\right)}{p_{0}^{k} \log x \log L_{k}} \\
& \asymp \frac{x}{L_{k} \log x \log L_{k}} .
\end{aligned}
$$

We have

$$
\begin{aligned}
\mid\left\{w \leq x / p_{0}^{k}:\right. & \left.: w \in \mathcal{D}_{p_{0}^{k}}, P^{-}(w)>L_{k}, \operatorname{gcd}(h, w)>1\right\} \mid \\
& \leq \sum_{\substack{p \mid h \\
p>L_{k}}}\left|\left\{w \leq x / p_{0}^{k}: w \in \mathcal{D}_{p_{0}^{k}}, P^{-}(w)>L_{k}, p \mid w\right\}\right| \\
& \ll \sum_{\substack{p \mid h \\
L_{k}<p \leq 2 L_{k}}} 1+\sum_{\substack{p \mid h \\
p>L_{k}}} \frac{x \log p}{L_{k} p \log x \log L_{k}},
\end{aligned}
$$

by Lemma 4, since $\log \left(p p_{0}^{k}\right) \ll \log p$ for $p>L_{k}$. The sum of 1 is clearly $\leq L_{k} \leq(x / 2)^{1 / 5}$. The second statement of the lemma now follows with the smallest $k \in \mathcal{K}$ such that $L_{k} \geq L$.

Since $h$ has at most $\log h / \log L_{k}$ prime factors $>L_{k}$, the last sum above is

$$
\ll \frac{\log h}{\log L_{k}} \cdot \frac{x}{L_{k} \log L_{k} \log x} \cdot \frac{\log L_{k}}{L_{k}}=\frac{x \log h}{L_{k}^{2} \log L_{k} \log x} .
$$

We need this to be $<x /\left(C L_{k} \log x \log L_{k}\right)$ for some sufficiently large constant $C>0$, that is, $L_{k} \geq C \log (2 h)$. The first statement of the lemma now follows with the smallest such $k \in \mathcal{K}$.

\section{The Lower Bound of Theorem 1}

Let $h$ be a fixed integer that is not a multiple of $\prod_{p \leq \theta(1)} p$. Let $\delta=1 / \log _{2} x$ and define

$$
\mathcal{Q}=\left\{q \in\left(x^{1 / 2-\delta}, x^{1 / 2} / \log ^{10} x\right]: \operatorname{gcd}(q, h)=1, q \in \mathcal{B}_{\theta}\right\} .
$$

Let $\mathcal{N}_{h}(x)$ denote the set of pairs $(q, m)$ with $q \in \mathcal{Q}, q m+h \leq x$, and $q m+h$ prime, and let $N_{h}(x)=\left|\mathcal{N}_{h}(x)\right|$. Thus,

$$
N_{h}(x)=\sum_{q \in \mathcal{Q}} \pi(x ; q, h)
$$

Now, by the Bombieri-Vinogradov theorem, see [21, p. 403], we have

$$
\sum_{q \in \mathcal{Q}}\left|\pi(x ; q, h)-\frac{\pi(x)}{\varphi(q)}\right| \ll \frac{x}{\log ^{6} x} .
$$


Thus,

$$
N_{h}(x)=\sum_{q \in \mathcal{Q}} \pi(x ; q, h)=\sum_{q \in \mathcal{Q}} \frac{\pi(x)}{\varphi(q)}+O\left(\frac{x}{\log ^{6} x}\right) .
$$

Further, using Lemma 5, we have

$$
\sum_{q \in \mathcal{Q}} \frac{1}{\varphi(q)} \geq \sum_{q \in \mathcal{Q}} \frac{1}{q} \gg_{h} \delta
$$

We conclude that

$$
N_{h}(x) \gg_{h} \delta x / \log x .
$$

Let $\mathcal{N}_{h, 1}(x)$ denote the set of those pairs $(q, m)$ in $\mathcal{N}_{h}(x)$ with $x^{\delta}<$ $P^{+}(m)<x^{1 / 2-\delta}$.

Lemma 6. We have $\left|\mathcal{N}_{h, 1}(x)\right|=\left|\mathcal{N}_{h}(x)\right|+O\left(\delta^{2} x / \log x\right)$,

Proof. Let $q \in \mathcal{Q}$. The number of integers $m \leq(x-h) / q$ with $P^{+}(m) \leq$ $x^{\delta}$ is $\ll(x-h) /\left(q \log ^{10} x\right)$, see [21, Lem. III.5.19], and so such numbers $m$ are negligible. For $m=r k$, where $r=P^{+}(m) \geq x^{1 / 2-\delta}$, we have $k \leq x^{2 \delta}$. Thus, the number of such pairs $(q, r k)$ is at most

$$
\sum_{q \in \mathcal{Q}} \sum_{k \leq x^{2 \delta}} \sum_{\substack{r \leq(x-h) / q k \\ r \text { prime } \\ q r k+h \text { prime }}} 1
$$

The inner sum, by Lemma 1, is $\ll_{h} x /\left(\varphi(q) \varphi(k) \log ^{2} x\right)$. Summing on $k$ gives us $\ll_{h} \delta x /(\varphi(q) \log x)$, and then summing on $q$ gives us $\ll_{h}$ $\delta^{2} x / \log x$, using $q / \varphi(q) \ll \sigma(q) / q$, Corollary 5 , and partial summation. This concludes the proof.

Corollary 6. For a pair $(q, m)$ in $\mathcal{N}_{h, 1}(x)$ we have $q m \in \mathcal{B}_{\theta}$.

Proof. Since $P^{+}(m)<x^{1 / 2-\delta}<q$, it follows from Lemma 2 that $q m \in$ $\mathcal{B}_{\theta}$.

Let $v_{2}(n)$ denote the number of factors 2 in the prime factorization of $n$ and let $\Omega(n)$ denote the total number of prime factors of $n$, counted with multiplicity. Let $\varepsilon>0$ be arbitrarily small but fixed. Let $\mathcal{N}_{h, 2}(x)$ denote the set of pairs $(q, m) \in \mathcal{N}_{h, 1}(x)$ with

$$
\Omega(m) \leq I:=\left\lfloor(1+\varepsilon) \log _{2} x\right\rfloor \text { and } v_{2}(m) \leq 4 \log _{3} x .
$$

Lemma 7. We have

$$
\left|\mathcal{N}_{h, 2}(x)\right|=\left|\mathcal{N}_{h}(x)\right|+O_{h}\left(\delta^{2} x / \log x\right) .
$$


Proof. Assume $(q, m) \in \mathcal{N}_{h, 1}(x)$. Let $r=P^{+}(m)$, so that $r>x^{\delta}$, and write $m=r k$. If $(q, m) \notin \mathcal{N}_{h, 2}(x)$ then either $\Omega(k)>I-1$ or $v_{2}(k)>4 \log _{3} x$. For a given number $k$, the number of primes $r \leq(x-$ $h) / q k$ with $q r k+h$ prime is, by Lemma 1, $\ll_{h} x /\left(\varphi(q) \varphi(k) \log ^{2}(x / q k)\right)$. Summing this expression over $k$ with $v_{2}(k)>4 \log _{3} x$ and $q \in \mathcal{Q}$, it is $\ll_{h} \delta^{2} x / \log x$, since $2^{-4 \log _{3} x}<\delta^{2}$. We now wish to consider the case when $\Omega(k)>I-1$. Following a standard theme (see Exercises 04 and 05 in [6]) we have uniformly for each real number $z$ with $1<z<2$ that

$$
\sum_{n \leq x} \frac{z^{\Omega(n)}}{\varphi(n)} \ll \frac{1}{2-z}(\log x)^{z} .
$$

Applying this with $z=1+\varepsilon$, we have

$$
\sum_{\substack{k \leq x^{1 / 2} \\ \Omega(k)>I-1}} \frac{1}{\varphi(k)} \leq z^{-I+1} \sum_{k \leq x^{1 / 2}} \frac{z^{\Omega(k)}}{\varphi(k)} \ll(\log x)^{1+\varepsilon-(1+\varepsilon) \log (1+\varepsilon)} .
$$

This last expression is of the form $(\log x)^{1-\eta}$, where $\eta>0$ depends on the choice of $\varepsilon$. Thus, the number of pairs $(q, m)$ in this case is $\ll_{h} \delta x /(\log x)^{1+\eta}$, which is negligible.

Let $\Omega_{3}(n)=\Omega\left(n / v_{2}(n)\right)$ denote the number of odd prime factors of $n$ counted with multiplicity, and let $\mathcal{N}_{h, 3}$ denote the number of pairs $(q, m) \in \mathcal{N}_{h, 2}$ with $\Omega_{3}(q) \leq J:=\left\lfloor(e+\varepsilon) \log _{2} x\right\rfloor$.

Lemma 8. We have $\left|\mathcal{N}_{h, 3}(x)\right|=\left|\mathcal{N}_{h}(x)\right|+O_{h}\left(\delta^{2} x / \log x\right)$.

Proof. By the same method that gives (9), we have

$$
\sum_{n \leq x} \frac{z^{\Omega_{3}(n)}}{\varphi(n)} \ll \frac{1}{3-z}(\log x)^{z},
$$

uniformly for $1<z<3$. Assuming that $\varepsilon$ is small enough that $z=$ $e+\varepsilon<3$, we have

$$
\sum_{\substack{q \in \mathcal{Q} \\ \Omega_{3}(q)>J}} \frac{1}{\varphi(q)} \leq \sum_{\substack{q \leq x^{1 / 2} \\ \Omega_{3}(q)>J}} \frac{1}{\varphi(q)} \leq z^{-J} \sum_{\substack{q x^{1 / 2}}} \frac{z^{\Omega_{3}(q)}}{\varphi(q)} \ll(\log x)^{z-(e+\varepsilon) \log z} .
$$

Since $z-(e+\varepsilon) \log z=-\eta<0$, where $\eta$ depends on the choice of $\varepsilon$, this calculation shows that those pairs with $\Omega_{3}(q)>J$ are negligible.

Let $K=\left\lfloor 4 \log _{3} x\right\rfloor+1$. For a given pair $(q, m) \in \mathcal{N}_{h, 3}(x)$, we count the number of pairs $\left(q^{\prime}, m^{\prime}\right) \in \mathcal{N}_{h, 3}(x)$ with $q^{\prime} m^{\prime}=q m$. The pair $\left(q^{\prime}, m^{\prime}\right)$ is determined by $(q, m)$ and $m^{\prime}$, so all we need to do is count 
the number of divisors $d$ of $q m$ with $\Omega(d) \leq I$ and $v_{2}(d)<K$. This count is at most

$$
K \sum_{i \leq I}\left(\begin{array}{c}
I+J \\
i
\end{array}\right) \ll K\left(\begin{array}{c}
I+J \\
I
\end{array}\right)
$$

Stirling's formula shows that

$$
K\left(\begin{array}{c}
I+J \\
I
\end{array}\right) \ll(\log x)^{\alpha+\eta} \log _{3} x
$$

where $\alpha=(e+1) \log (e+1)-e \log e=2.16479 \ldots$ and $\eta \rightarrow 0$ as $\varepsilon \rightarrow 0$. It follows from (8) and Lemma 8 that

$$
S_{h}(x) \gg \frac{\delta x}{\log x} \cdot \frac{1}{(\log x)^{\alpha+\eta} \log _{3} x} \gg \frac{x}{(\log x)^{1+\alpha+2 \eta}}=\frac{x}{(\log x)^{3.16479 \ldots+2 \eta}} .
$$

Remark 2. The proof of the lower bound of Theorem 1 would be somewhat simpler if instead of the Bombieri-Vinogradov theorem we had used a very new result of Maynard 9]. With the choice of parameters $\delta=0.02, \eta=0.001$ in his Corollary 1.2 , one has for the set $\mathcal{Q}$ of integers $q \leq x^{0.52}$ with a divisor in $\left(x^{0.041}, x^{0.071}\right)$ that

$$
\sum_{\substack{q \in \mathcal{Q} \\ \operatorname{gcd}(q, a)=1}}\left|\pi(x ; q, a)-\frac{\pi(x)}{\varphi(q)}\right| \ll_{a, A} \frac{x}{\log ^{A} x},
$$

for any fixed integer $a \neq 0$ and any positive $A$. We note that all of the members of $\mathcal{B}_{\theta} \cap\left(x^{0.041}, x^{0.52}\right]$ are in $\mathcal{Q}$.

\section{Proof of Theorem 2}

Let $h$ be an integer in $(x / 2, x]$ that is not a multiple of $\prod_{p \leq \theta(1)} p$. Define

$$
\mathcal{D}=\left\{q \in \mathcal{B}_{\theta} \cap\left(x^{1 / 2-\delta}, x^{1 / 2} / \log ^{10} x\right]: \operatorname{gcd}(q, h)=1\right\} .
$$

By Lemma 5,

$$
|\mathcal{D}| \gg \frac{x^{1 / 2}}{\log ^{12} x \log \log x} .
$$

For each $q \in \mathcal{D}$, if $p \leq x / 2<h$, where $p$ is a prime that satisfies $p \equiv h \bmod q$, then $p=\bar{h}-q m$ for some $m \in \mathbb{N}$. Let $M_{h}(x)$ denote the number of pairs $(p, q)$ with $p$ prime, $p \leq x / 2, p \equiv h \bmod q$ and $q \in \mathcal{D}$. As in Section 4, we have

$$
M_{h}(x)=\sum_{q \in \mathcal{D}} \pi(x / 2 ; q, h)=\sum_{q \in \mathcal{D}} \frac{\pi(x / 2)}{\varphi(q)}+O\left(\frac{x}{\log ^{6} x}\right) .
$$


From (11), we have

$$
F:=\sum_{q \in \mathcal{D}} \frac{1}{\varphi(q)} \geq \sum_{q \in \mathcal{D}} \frac{1}{q} \geq \frac{|\mathcal{D}|}{x^{1 / 2} / \log ^{10} x} \gg \frac{1}{\log ^{2} x \log \log x} .
$$

We conclude that

$$
M_{h}(x) \gg F \frac{x}{\log x} \gg \frac{x}{\log ^{3} x \log \log x} .
$$

We claim that most of the pairs $(p, q)$ counted in $M_{h}(x)$ are such that $q m=h-p \in \mathcal{B}_{\theta}$. Since $q>x^{1 / 2-\delta}$ and $q m<h \leq x$, we have $m \leq x^{1 / 2+\delta}$. If $P^{+}(m) \leq x^{1 / 2-\delta}$, then $P^{+}(m)<q$ and $m q \in \mathcal{B}_{\theta}$. If $P^{+}(m)>x^{1 / 2-\delta}$, write $r=P^{+}(m)>x^{1 / 2-\delta}$ and $m=r a$ with $a<x^{2 \delta}$. Given $a$ and $q$, the number of primes $r<x /(a q)$ with $h-a q r$ prime is

$$
\ll \frac{h x}{\varphi(h) \varphi(q) \varphi(a) \log ^{2} x},
$$

by Lemma 1, We have $h / \varphi(h) \ll \log \log x$ and

$$
\sum_{a<x^{2 \delta}} \frac{1}{\varphi(a)} \ll \delta \log x
$$

Thus, summing (13) over $q \in \mathcal{D}$ and $a<x^{2 \delta}$ amounts to

$$
\ll F \frac{x \delta \log \log x}{\log x}=o\left(F \frac{x}{\log x}\right),
$$

since $\delta=1 /(\log \log x)^{2}$. By (12), the number of pairs $(p, q)$ with $h=$ $p+q m, p$ prime and $q m \in \mathcal{B}_{\theta}$ is

$$
\gg F \frac{x}{\log x} \gg \frac{x}{\log ^{3} x \log \log x},
$$

which is at least 1 when $x$ is sufficiently large. This completes the proof of Theorem 2 .

5.1. Checking Margenstern's conjecture numerically. For positive coprime integers $u, v$, let $p(u, v)$ be the least prime $p \equiv u(\bmod v)$, and let $M(v)=\max _{\operatorname{gcd}(u, v)=1} p(u, v)$. For example, $M(8)=17$, since $p(1,8)=17, p(3,8)=3, p(5,8)=5$, and $p(7,8)=7$.

Lemma 9. Suppose that a is a positive integer with $M\left(2^{a}\right)<2^{2 a+1}$. Then every odd number $n \in\left(M\left(2^{a}\right), 2^{2 a+1}\right)$ is the sum of a prime and a practical number.

Proof. For each odd $n \in\left(M\left(2^{a}\right), 2^{2 a+1}\right)$ let $q=n-p\left(n, 2^{a}\right)$. Note that $0<q<2^{2 a+1}$ and $2^{a} \mid q$. Since $2^{a}$ is practical and $\sigma\left(2^{a}\right)+1=$ $2^{a+1}>q / 2^{a}$, it follows that $q$ is practical. Thus, $n=q+p\left(n, 2^{a}\right)$ is a representation of $n$ as the sum of a prime and a practical. 
Note that the condition in Lemma 9 that $M\left(2^{a}\right)<2^{2 a+1}$ is not guaranteed by any known result in analytic number theory. We do know that $M\left(2^{a}\right) \leq 2^{O(a)}$ with a fairly modest $O$-constant, but we are not close to proving the condition in the lemma. (Heuristically, we should have $M\left(2^{a}\right)=O\left(2^{a} a^{2}\right)$.) For a given numerical value of $a$, one might actually compute the exact value of $M\left(2^{a}\right)$. And if $\mathrm{f}$ it is smaller than $2^{2 a+1}$, we have verified Margenstern's conjecture for the interval $\left(M\left(2^{a}\right), 2^{2 a+1}\right)$. For example, since $M\left(2^{3}\right)=17$, we automatically have the conjecture for odd numbers in the interval $(17,128)$.

We have computed that $M\left(2^{23}\right)=997,427,777$. This number is less than $2^{47}$, in fact, it is less than $10^{9}$. Thus, Margenstern's conjecture holds for all odd numbers (greater than 1) up to $2^{47}$. Moreover, since $M\left(2^{35}\right)=9,968,601,716,713<2^{47}$, the conjecture holds up to $2^{71}$. It would not be difficult to push this calculation further.

\section{The Upper Bound in TheOREMs 3 AND 4}

For a natural number $n$, a divisor $d$ of $n$ is said to be initial if $P^{+}(d) \leq P^{-}(n / d)$. Let $I_{y}(n)$ be the largest initial divisor of $n$ with $d \leq y$. Note that if $n \in \mathcal{B}_{\theta}$, then $I_{y}(n) \in \mathcal{B}_{\theta}$ for all $y$.

Assume $n \leq x$ and $n, n+h \in \mathcal{B}_{\theta}$. Let $q=I_{x^{1 / 3}}(n), q^{\prime}=I_{x^{1 / 3}}(n+h)$. Since $n, n+h \in \mathcal{B}_{\theta}$ and $\theta(n)=n^{1+o(1)}$, we may assume that $q, q^{\prime} \in$ $\left[x^{1 / 7}, x^{1 / 3}\right]$. Write $n=q m$ and $n+h=q^{\prime} m^{\prime}$. We have $q, q^{\prime} \in \mathcal{B}_{\theta}$ and $P^{-}(m) \geq P^{+}(q)=: r, P^{-}\left(m^{\prime}\right) \geq P^{+}\left(q^{\prime}\right)=: r^{\prime}$. Given $q, q^{\prime} \in \mathcal{B}_{\theta}$ with $d=\operatorname{gcd}\left(q, q^{\prime}\right)$, we need $m, m^{\prime}$ such that $q^{\prime} m^{\prime}-q m=h$. This equation only has solutions if $d \mid h$, in which case all solutions have the form

$$
m=m_{0}+j q^{\prime} / d, \quad m^{\prime}=m_{0}^{\prime}+j q / d, \quad j \in \mathbb{Z} .
$$

If $m_{0}, m_{0}^{\prime}$ are the smallest positive solutions to $q^{\prime} m^{\prime}-q m=h$, then $1 \leq n=m q \leq x$ implies $0 \leq j \leq d x / q q^{\prime} \leq h x / q q^{\prime}$. Let

$$
\mathcal{A}=\left\{\left(m_{0}+j q^{\prime} / d\right)\left(m_{0}^{\prime}+j q / d\right): 0 \leq j \leq h x / q q^{\prime}\right\},
$$

and let $S(\mathcal{A})$ be the number of elements of $\mathcal{A}$ remaining after removing all products $m m^{\prime}$, where either $m$ is a multiple of a prime $p<r, p \nmid h q q^{\prime}$, or $m^{\prime}$ is a multiple of a prime $p<r^{\prime}, p \nmid h q q^{\prime}$. For each prime $p \nmid h q q^{\prime}$, each of the conditions $p \mid m$ and $p \mid m^{\prime}$ is equivalent to $j$ belonging to a unique residue class modulo $p$ (because $p \nmid q q^{\prime}$ ), and those two residue classes are distinct (because $p \nmid h$ ). Selberg's sieve [3, Prop. 7.3 and Thm. 7.14] shows that

$$
S(\mathcal{A}) \ll \frac{h x / q q^{\prime}}{\log r \log r^{\prime}}\left(\frac{h q q^{\prime}}{\varphi\left(h q q^{\prime}\right)}\right)^{2} \ll_{h} \frac{x q q^{\prime}}{\varphi(q)^{2} \varphi\left(q^{\prime}\right)^{2} \log P^{+}(q) \log P^{+}\left(q^{\prime}\right)} .
$$


Summing this estimate over $q, q^{\prime} \in\left[x^{1 / 7}, x^{1 / 3}\right] \cap \mathcal{B}_{\theta}$, the upper bound in Theorem 3 follows from Lemma 10 with $\alpha=2$.

This argument generalizes naturally to yield Theorem 4; For $1 \leq$ $i \leq k$, let $n+h_{i}=m_{i} q_{i} \in \mathcal{B}_{\theta}$, where $q_{i}=I_{x^{1 /(k+1)}}\left(n+h_{i}\right)$, so that $q_{i} \in \mathcal{B}_{\theta} \cap\left[x^{1 /(2 k+3)}, x^{1 /(k+1)}\right]$. One finds that if $\operatorname{gcd}\left(q_{i}, q_{l}\right) \mid\left(h_{l}-h_{i}\right)$, for $1 \leq i<l \leq k$, then

$$
m_{i}=m_{i, 0}+j \operatorname{lcm}\left(q_{1}, \ldots, q_{k}\right) / q_{i} \quad(1 \leq i \leq k),
$$

where $0 \leq j \leq x / \operatorname{lcm}\left(q_{1}, \ldots, q_{k}\right) \leq \frac{x}{q_{1} \ldots q_{k}} \prod_{1 \leq i<l \leq k}\left(h_{l}-h_{i}\right)$. Eliminating values of $j$ for which $p \mid m_{i}$, where $p<P^{+}\left(q_{i}\right), p \nmid \prod_{i \leq k} q_{i}$ and $p \nmid$ $\prod_{1 \leq i<l \leq k}\left(h_{l}-h_{i}\right)$, we find that

$$
S(\mathcal{A}) \ll_{h_{1}, \ldots, h_{k}} x \prod_{i=1}^{k} \frac{q_{i}^{k-1}}{\varphi\left(q_{i}\right)^{k} \log P^{+}\left(q_{i}\right)} .
$$

Theorem 4 now follows from Lemma 10 with $\alpha=k$.

Lemma 10. Let $\alpha \in \mathbb{R}$. Assume (2) and $\theta(n) \ll n l(n)$ for $n \geq 1$. We have

$$
\sum_{q \geq x, q \in \mathcal{B}_{\theta}} \frac{q^{\alpha-1}}{\varphi(q)^{\alpha} \log P^{+}(q)} \ll_{\alpha} \frac{1}{\log x} .
$$

Proof. It suffices to estimate the sum restricted to $q \in I:=\left[x, x^{4 / 3}\right]$. We write $q=m r$, where $r=P^{+}(q)$. Note that $q \in \mathcal{B}_{\theta} \cap I$ and $\theta(n)<n^{1+o(1)}$ implies that $r \leq x^{3 / 4}$. We have

$$
\sum_{q \in \mathcal{B}_{\theta} \cap I} \frac{q^{\alpha-1}}{\varphi(q)^{\alpha} \log P^{+}(q)} \ll \sum_{r \leq x^{3 / 4}} \frac{1}{r \log r} \sum_{\substack{m \in \mathcal{B}_{\theta} \cap(I / r) \\ P^{+}(m) \leq r}}\left(\frac{m}{\varphi(m)}\right)^{\alpha} \frac{1}{m} .
$$

Since $m / \varphi(m) \ll \sigma(m) / m$, partial summation and Corollary 4 applied to the inner sum shows that the last expression is

$$
\ll_{\alpha} \sum_{r \leq x^{3 / 4}} \frac{1}{r \log r} \cdot \frac{\log r}{\log x} \exp \left(-\frac{\log x}{3 \log r}\right) \ll \frac{1}{\log x},
$$

by the prime number theorem.

\section{The Lower Bound in Theorem 3}

Lemma 11. Assume (2) and $\theta(n) \ll n l(n)$ for $n \geq 1$. For $L \geq 1$ and $x \geq 1$, we have

$$
\sum_{\substack{n \in \mathcal{B}_{\theta} \\ n \leq x}} \sum_{\substack{p>n \\ p>L}} \frac{\log p}{p} \ll \frac{x \log (2 L)}{L \log (2 x)} .
$$


Proof. As in the proof of Corollary 4 ,

$$
\begin{aligned}
\sum_{\substack{n \in \mathcal{B}_{\theta} \\
n \leq x}} \sum_{\substack{p \mid n \\
p>L}} \frac{\log p}{p} & =\sum_{\substack{L<p<x^{2 / 3} \\
p}} \frac{\log p}{p} \sum_{\substack{m p \in \mathcal{B}_{\theta} \\
m \leq x / p}} 1 \leq \sum_{L<p<x^{2 / 3}} \frac{\log p}{p} \sum_{\substack{m \in \mathcal{B}_{\theta p} \\
m \leq x / p}} 1 \\
& \ll \sum_{L<p<x^{2 / 3}} \frac{\log p}{p} \cdot \frac{x \log p}{p \log (2 x)} \ll \frac{x \log (2 L)}{L \log (2 x)},
\end{aligned}
$$

by Proposition 1 and the prime number theorem.

Say a pair $n_{1}, n_{2} \in \mathcal{B}_{\theta}$ is $h$ - $\varepsilon$-special if $\operatorname{gcd}\left(n_{1}, n_{2}\right)=h$ and $\Omega_{3}\left(n_{i}\right) \leq$ $(e+\varepsilon) \log _{2} n_{i}$ for $i=1,2$.

Lemma 12. Assume (2) and $n \leq \theta(n) \ll n l(n)$ for $n \geq 1$. For $h \geq 1$ satisfying (5) and $0<\varepsilon<1$, the number of $h$ - $\varepsilon$-special pairs $n_{1}, n_{2} \in \mathcal{B}_{\theta}$ with $N / 3<n_{1}, n_{2}<N$ and $v_{2}\left(n_{1}\right), v_{2}\left(n_{2}\right) \leq C$, where $C$ is some number depending only on $h$, is $\gg_{h, \varepsilon} N^{2} / \log ^{2} N$.

Proof. Write $h=2^{a} 3^{b} h^{\prime}$, where $P^{-}\left(h^{\prime}\right)>3, a, b \geq 0$, but assume that $a \geq 1$ or $a \geq 2$, according to the two cases in (5). We consider $n_{1} \in \mathcal{B}_{\theta}$ of the form

$$
n_{1}=2^{a+k} 3^{b} h^{\prime} n_{1}^{\prime}=2^{k} h n_{1}^{\prime}
$$

where $P^{-}\left(n_{1}^{\prime}\right)>\max \left\{3, P^{+}(h)\right\}=: p$ and $2^{k}>2 p$. Since $\theta(n) \geq n$, the number of such $n_{1}$ with $N / 2<n_{1} \leq N$ is at least

$$
\left|\left\{\frac{N}{h 2^{k+1}}<n_{1}^{\prime} \leq \frac{N}{h 2^{k}}: n_{1}^{\prime} \in \mathcal{D}_{h 2^{k}}, P^{-}\left(n_{1}^{\prime}\right)>p\right\}\right| \asymp_{h} \frac{N}{\log N},
$$

by Lemma 3, for a suitable $k$ with $2^{k}>2 p>2^{k+O(1)}$. In particular, $v_{2}\left(n_{1}\right) \ll_{h} 1$.

As in the proof of the lower bound of Theorem 11, we can remove those $n_{1}$ with $\Omega_{3}\left(n_{1}\right)>(e+\varepsilon) \log _{2} n_{1}$ without affecting (14). This follows from an estimate analogous to (10):

$$
\sum_{n \leq x} z^{\Omega_{3}(n)} \ll \frac{x}{3-z} \log ^{z-1} x
$$

uniformly for $1<z<3$ (cf. [21, Exercise 217(b)]).

Let $\eta>0$ be an arbitrary constant. Lemma [1] shows that we can choose a sufficiently large constant $L=L(\eta)$ such that removing those $n_{1}$ for which

$$
\sum_{\substack{p \mid n_{1} \\ p>L}} \frac{\log p}{p}>\eta
$$


will not affect (14). For each of the $\asymp_{h, \varepsilon} N / \log N$ values of $n_{1}$ that remain, consider $n_{2} \in \mathcal{B}_{\theta}$ of the form

$$
n_{2}=2^{a} 3^{b+j} h^{\prime} n_{2}^{\prime}=3^{j} h n_{2}^{\prime},
$$

where $\operatorname{gcd}\left(n_{2}^{\prime}, 2 n_{1}^{\prime}\right)=1$, and $j$ is the smallest integer with $3^{j}>p$. Given $n_{1}$, the number of such $n_{2} \leq N$ is at least

$$
\sum_{\substack{N / h 3^{j+1}<n_{2}^{\prime} \leq N / h 3^{j} \\ n_{2}^{\prime} \in \mathcal{D}_{h 3}^{j} \\ \operatorname{gcd}\left(n_{2}^{\prime}, 2 n_{1}^{\prime}\right)=1}} 1 \gg_{h} \frac{N}{\log (N L) \log (2 L)} \gg \frac{N}{\log N},
$$

by Lemma 5 with $p_{0}=3$. As with $n_{1}$, this estimate is unchanged if we remove those $n_{2}$ with $\Omega\left(n_{2}\right)>(e+\varepsilon) \log _{2} n_{2}$. Further, $v_{2}\left(n_{2}\right)=$ $v_{2}(h) \ll_{h} 1$.

Let $N=\sqrt{x h}$. Suppose $a, a^{\prime} \in \mathcal{B}_{\theta} \cap(N / 3, N]$ is an $h$ - $\varepsilon$-special pair, with $v_{2}(a), v_{2}\left(a^{\prime}\right) \leq C$, where $C=C(h)$ is as in Lemma 12. For each such pair $\left\{a, a^{\prime}\right\}$, there is a unique pair $\left\{b, b^{\prime}\right\}$ such that $a b-a^{\prime} b^{\prime}=h$ and $1 \leq b \leq a^{\prime} / h, 1 \leq b^{\prime} \leq a / h$. We have $a b, a^{\prime} b^{\prime} \leq a a^{\prime} / h \leq x$. Now $b, b^{\prime} \leq \sqrt{x / h}<3 a / h, 3 a^{\prime} / h$, so $a b, a^{\prime} b^{\prime} \in \mathcal{B}_{\theta}$ by the assumption on $\theta$. By Lemma 12, it would seem we have created $\gg_{h, \varepsilon} x / \log ^{2} x$ pairs $\left\{a b, a^{\prime} b^{\prime}\right\} \subset \mathcal{B}_{\theta} \cap[1, x]$ with $a b-a^{\prime} b^{\prime}=h$, but we have to check for possible multiple representations.

Note that in a graph of average degree $\geq d$, there is an induced subgraph of minimum degree $\geq d / 2$. This folklore result can be proved by induction on $d$, see [1]. (Also see [7, Prop. 3] for a somewhat sharper version.) We apply this to the graph on members of $\mathcal{B}_{\theta} \cap(N / 3, N]$, where two integers are connected by an edge if they form an $h$ - $\varepsilon$-special pair. From Lemma 12 the average degree in this graph is $\gg N / \log N$, so there is a subgraph $G$ of minimum degree $\gg N / \log N$.

We use this to say something about $\Omega_{3}(b), \Omega_{3}\left(b^{\prime}\right)$. For edges $\left(a, a^{\prime}\right)$ in $G$, note that for any residue class mod $a^{\prime}$ there are at most 2 choices for $a$, and similarly for any residue class $\bmod a$ there are at most 2 choices for $a^{\prime}$. For $\left(a, a^{\prime}\right)$ with corresponding pair $\left(b, b^{\prime}\right)$ as above, let $f\left(a, a^{\prime}\right)=b$ and $g\left(a, a^{\prime}\right)=b^{\prime}$. For each fixed $a^{\prime}$ the function $f$ is at most two-to-one in the variable $a$, since $(a / h) b \equiv 1\left(\bmod a^{\prime} / h\right)$ and $b \leq a^{\prime} / h$. Similarly, for each fixed $a$, the function $g\left(a, a^{\prime}\right)=b^{\prime}$ is at most two-to-one in the variable $a^{\prime}$. Thus, for each fixed $a^{\prime}$ there are $\gg N / \log N$ distinct values of $b$ and for each fixed $a$ there are $\gg N / \log N$ distinct values of $b^{\prime}$. Now $b, b^{\prime} \leq N$ and as we have seen, the number of integers $n \leq N$ with $\Omega_{3}(n)>(e+\varepsilon) \log _{2} x$ is $o(N / \log N)$. So, by possibly discarding $o\left(x / \log ^{2} x\right)$ pairs $\left(a, a^{\prime}\right)$, we may assume that the corresponding pair $\left(b, b^{\prime}\right)$ satisfies $\Omega_{3}(b), \Omega_{3}\left(b^{\prime}\right) \leq(e+\varepsilon) \log _{2} x$. 
The numbers $a b$ and $a^{\prime} b^{\prime}$ might arise from many different pairs $\left(a, a^{\prime}\right)$. However, we have $\Omega_{3}(a b), \Omega_{3}\left(a^{\prime} b^{\prime}\right) \leq 2(e+\varepsilon) \log _{2} x$, so the number of odd divisor pairs of $a b, a^{\prime} b^{\prime}$ is

$$
\leq 2^{4(e+\varepsilon) \log _{2} x}=(\log x)^{4(e+\varepsilon) \log 2} .
$$

Since $v_{2}(a), v_{2}\left(a^{\prime}\right) \ll_{h} 1$, there are $\gg_{h, \varepsilon} x /(\log x)^{2+4(e+\varepsilon) \log 2}$ pairs $n, n+$ $h \in \mathcal{B}_{\theta}$ with $n \leq x$. This completes the proof of the theorem.

Acknowledgments. We thank David Eppstein for informing us of [7] and Paul Pollack for [18].

\section{REFERENCES}

[1] M. DeVos, Graph theory lecture notes, www.sfu.ca/ mdevos/notes/graph/.

[2] P. Erdős, On a diophantine equation, Mat. Lapok 1 (1950), 192-210.

[3] J. Friedlander and H. Iwaniec, Opera de cribro. American Mathematical Society Colloquium Publications, 57, American Mathematical Society, Providence, 2010.

[4] V. Guo and A. Weingartner, The practicality of shifted primes, Integers $\mathbf{1 8}$ (2018), A93, 7 pp.

[5] R. K. Guy, Unsolved problems in number theory. Third ed. Springer-Verlag, New York, 2004.

[6] R. R. Hall and G. Tenenbaum, Divisors, Cambridge University Press, 1988.

[7] D. R. Lick and A. T. White, $k$-degenerate graphs, Canadian J. Math. 22 (1970), 1082-1096.

[8] M. Margenstern, Les nombres pratiques: théorie, observations et conjectures, J. Number Theory 37 (1991), 1-36.

[9] J. Maynard, Primes in arithmetic progressions to large moduli I: fixed residue classes, arXiv:2006.06572v1 [math.NT] 11 Jun 2020.

[10] G. Melfi, On two conjectures about practical numbers, J. Number Theory $\mathbf{5 6}$ (1996), 205-210.

[11] G. Melfi, A note on twin practical numbers, Matematiche (Catania) 57 (2002), 111-117 (2004).

[12] H. Riesel and R. Vaughan, On sums of primes, Arkiv. för Mat. 21 (1983), 45-74.

[13] E. Saias, Entiers à diviseurs denses 1, J. Number Theory 62 (1997), 163-191.

[14] E. Saias, Entiers à diviseurs denses 2, J. Number Theory 86 (2001), 39-49.

[15] W. Sierpinski, Sur une propriété des nombres naturels, Ann. Mat. Pura Appl. (4) 39 (1955), 69-74.

[16] A. K. Srinivasan, Practical numbers, Current Sci. 17 (1948), 179-180.

[17] B. M. Stewart, Sums of distinct divisors, Amer. J. Math. 76 (1954), 779-785.

[18] Z.-W. Sun, Write $2 n+1=p+q$ with $p$ prime and $q$ practical, https ://mathoverflow.net/questions/360288/

[19] Z.-W. Sun, Conjectures on representations involving primes, in Combinatorial and additive number theory II, M. B. Nathanson, ed., Springer PROMS 220, New York, 2018, pp. 279-310.

[20] G. Tenenbaum, Sur un problème de crible et ses applications, Ann. Sci. École Norm. Sup. (4) 19 (1986), 1-30. 
[21] G. Tenenbaum, Introduction to analytic and probabilistic number theory. Third ed. Graduate Studies in Mathematics, Vol. 163, American Math. Soc., Providence, 2015.

[22] A. Weingartner, Integers with dense divisors, J. Number Theory 108 (2004), $1-17$.

[23] A. Weingartner, Practical numbers and the distribution of divisors, Q. J. Math. 66 (2015), 743-758.

[24] A. Weingartner, The constant factor in the asymptotic for practical numbers, Int. J. Number Theory 16 (2020), 629-638.

Mathematics Department, Dartmouth College, Hanover, NH 03784

Email address: carl.pomerance@dartmouth.edu

Department of Mathematics, Southern Utah University, Cedar City, UT 84720

Email address: weingartner@suu.edu 